11

\title{
Экситонный фазовый переход моттовского типа металл-диэлектрик в сжатом кальции
}

\author{
(C) Т.О. Воронкова, А.М. Сарры, М.Ф. Сарры , С.Г. Скидан
}

Российский федеральный ядерный центр-Всероссийский научно-исследовательский институт экспериментальной физики, Саров, Россия

ฯ E-mail: sarry-vniief@yandex.ru

(Поступила в Редакцию 25 мая 2016 г.

В окончательной редакции 9 октября 2016 г.)

\begin{abstract}
Экспериментально установлено, что при статическом сжатии кристалла Са, находящегося при комнатной температуре, он претерпевает ряд структурных фазовых переходов: гранецетрированная кубическая $\rightarrow$ объемно центрированная кубическая $\rightarrow$ простая кубическая решетка. Было решено исследовать именно простую кубическую решетку (так как она является альтернантной решеткой) на возможность существования в ней еще и других (неструктурных) фазовых переходов, используя для этого модель Хаббарда для электронов с половинным заполнением $n s$-зон и предварительно преобразовав исходную электронную систему в электронно-дырочную систему с помощью известных операторов Шибы (применимых только к альтернантным решеткам). После такого преобразования в новой системе фермионов вместо прежнего отталкивания появилось притяжение между электронами и дырками. Элементарными возбуждениями этой новой системы являются связанные бозонные образования - экситоны. Эта фермионная система количественно исследовалась путем совместного использования метода уравнений движения и прямого алгебраического метода. Численным интегрированием полученных из первых принципов аналитически точных трансцендентных уравнений для альтернантных решеток (одно-, дву- и трехмерных) показано, что в системах двухсортных фермионов (электроны + дырки) действительно возможны и температурные фазовые превращения моттовского типа металл-изолятор. Более того, все эти кристаллы фактически оказываются экситонными изоляторами, что полностью согласуется с аналитически точными расчетами основного состояния одномерного кристалла (с половинным заполнением его зон), выполненными в работе Либа и Ву с целью обнаружить моттовский переход другого типа изолятор-металл.
\end{abstract}

DOI: 10.21883/FTT.2017.05.44386.217

\section{1. Введение}

В настоящей работе на основе модели Хаббарда [1] изучаются термодинамические свойства изотермически сжатого щелочно-земельного металла - кристалла Са с простой кубической (ПК) решеткой и одной невырожденной $n s$-зоной атомов $\left({ }_{40} \mathrm{Ca}^{20}\right)$, хотя известно, что обычные металлы при нормальных условиях не кристаллизуются в решетку этого типа. Однако при изменении внешних условий исходный тип решетки может измениться. Именно это и происходит с Са: имея в качестве исходной (т.е. при нормальных условиях) ГЦК-решетку, Са перекристаллизовывается в ПК-решетку при $300 \mathrm{~K}$ и внешнем статическом (изотермическом) давлении в интервале $32 \leq P \leq 80 \mathrm{GPa}$ (точнее, происходит ряд структурных фазовых переходов: $10^{5} \mathrm{~Pa} \leq$ ГЦК-решетка $\leq 19.5 \mathrm{GPa} \leq$ ОЦК-решетка $\leq 32 \mathrm{GPa} \leq$ ПК-решетка $\leq 80 \mathrm{GPa}$, см. работу [2] и ссылки в ней). Этот экспериментальный результат представляется несколько странным, поскольку при сжатии кристалла Са его коэффициент упаковки уменьшается со значения 0.74 для исходной ГЦК-решетки (здесь на элементарную ячейку кристалла приходится четыре атома) до значения 0.52 для сжатой ПК-решетки (здесь на элементарную кристаллическую ячейку приходится всего один атом), что составляет около $30 \%$.

ПК-решетка, являясь альтернантной решеткой, допускает использование своеобразных канонических преобразований с помощью операторов Шибы [3] (эти преобразования не меняют характер перестановочных соотношений новых операторов), позволяющих исходную односортную систему фермионов с внутренним отталкиванием превратить в двухсортную систему фермионов с притяжением между разными сортами фермионов, из которых в результате образуется система нейтральных бозонов - экситонов.

Цель настоящей работы состоит в численном изучении возможных решений уравнения для щели в спектре элементарных возбуждений полученной двухсортной системы фермионов, а также уравнения для температуры исчезновения щели - критической температуры.

\section{2. Однозонный гамильтониан Хаббарда и канонические преобразования Шибы}

Гамильтониан Хаббарда кристалла с фиксированной решеткой из атомов с одной невырожденной электрон- 
ной энергетической $n s$-зоной в представлении чисел заполнения одноэлектронных состояний имеет вид [1]

$H=\sum_{j j^{\prime} \sigma} t_{j j^{\prime}} \hat{C}_{j \sigma}^{+} \hat{C}_{j^{\prime} \sigma}+(U / 2) \sum_{j \sigma} \hat{n}_{j \sigma} \hat{n}_{j-\sigma}, \hat{n}_{j \sigma} \equiv \hat{C}_{j \sigma}^{+} \hat{C}_{j \sigma}$,

где суммирование производится по узлам $j, j^{\prime}$ решетки и значениям спинового числа $\sigma, t_{j j^{\prime}}-$ кинетическая энергия перескока электрона с узла $j$ на узел $j^{\prime}$.

Тогда канонические преобразования Шибы [3] исходных операторов имеют вид

$$
\begin{gathered}
\hat{C}_{j \uparrow}^{ \pm} \equiv \hat{p}_{j \uparrow}^{ \pm}, \quad \hat{C}_{j \downarrow}^{ \pm} \equiv \exp (\mp i \mathbf{q} j) \hat{h}_{j \uparrow}^{\mp}, \\
\mathbf{q}=\left(q_{x}, q_{y}, q_{z}\right)=(\pi / a)(1,1,1), \quad \mathbf{q} j \equiv \mathbf{q} \mathbf{R}_{j} .
\end{gathered}
$$

Здесь $\hat{p}_{j \uparrow}^{ \pm}$и $\hat{h}_{j \uparrow}^{\mp}-$ операторы рождения (уничтожения) электронов и дырок (индексы „Плюс“, „минус“"), $\mathbf{R}_{j}-$ радиус-вектор $j$-го узла.

Таким образом, в новой Ферми-системе будут фигурировать лишь электроны и дырки с одинаковым направлением спинов, а гамильтониан (1), записанный с помощью новых операторов (2), принимает вид

$$
\begin{aligned}
H= & \sum_{j j^{\prime}} t_{j j^{\prime}}\left(\hat{p}_{j \uparrow}^{+} \hat{p}_{j^{\prime} \uparrow}+\hat{h}_{j \uparrow}^{+} \hat{h}_{j^{\prime} \uparrow}\right) \\
& +U \sum_{j} \hat{p}_{j \uparrow}^{+} \hat{p}_{j \uparrow}-U \sum_{j} \hat{p}_{j \uparrow}^{+} \hat{p}_{j \uparrow} \hat{h}_{j \uparrow}^{+} \hat{h}_{j \uparrow} .
\end{aligned}
$$

Таким образом, отталкивающее взаимодействие $U$ в (1), присутствовавшее между электронами одного узла в исходной системе, превратилось в притягивающее взаимодействие между электронами и дырками узла в новой системе, в которой число электронов и дырок разное, но их спины теперь имеют одинаковое направление. Поэтому далее индекс спина у них опущен. Отметим, что это преобразование не является вполне частично-дырочным преобразованием, где состоянию $\mathbf{k}, \alpha, s_{z}$ электрона выше уровня Ферми $\varepsilon_{\mathrm{F}}$ соответствует состояние $-\mathbf{k}, \alpha,-s_{z}$ дырки ниже $\varepsilon_{\mathrm{F}}$, которое используется для описания слабовозбужденных электронных систем (здесь $\mathbf{k}-$ волновой вектор, $s_{z}-$ проекция спина, $\alpha-$ совокупность остальных квантовых чисел).

Поскольку при $\delta \equiv V_{0} / V=2$, т. е. при двукратном сжатии $(P \approx 68 \mathrm{GPa})$ при температуре $T=300 \mathrm{~K}$, кальций имеет устойчивую ПК-решетку, было решено изучить такой Са на предмет возможности существования в нем еще и другого типа фазовых переходов (ФП), например температурных.

Решеточные (канонические) преобразования [4] имеют вид

$$
\begin{aligned}
& \hat{p}_{\mathbf{k} \uparrow}^{ \pm}=\frac{1}{\sqrt{N}} \sum_{j} \hat{p}_{j \uparrow}^{ \pm} \exp \left(\mp i \mathbf{k} \mathbf{R}_{j}\right), \\
& \hat{p}_{j \uparrow}^{ \pm}=\frac{1}{\sqrt{N}} \sum_{\mathbf{k}} \hat{p}_{\mathbf{k} \uparrow}^{ \pm} \exp \left( \pm i \mathbf{k} \mathbf{R}_{j}\right) .
\end{aligned}
$$

Здесь суммирование по $j$ идет по всем $N$ узлам решетки, a значения $\mathbf{k}$ берутся лишь из первой зоны Бриллюэна.
Тогда унитарность $\left(\hat{A}^{+}=\hat{A}^{-1}\right)$ этих преобразований будет обеспечиваться равенствами

$$
\begin{aligned}
& \frac{1}{N} \sum_{j} \exp \left[i\left(\mathbf{k}-\mathbf{k}^{\prime}\right) \mathbf{R}_{j}\right]=\delta_{\mathbf{k k}^{\prime}}, \\
& \frac{1}{N} \sum_{\mathbf{k}} \exp \left[i\left(\mathbf{R}_{j}-\mathbf{R}_{j^{\prime}}\right) \mathbf{k}\right]=\delta_{j j^{\prime}},
\end{aligned}
$$

где $\delta_{j j^{\prime}}$ - дельта-символ Кронекера.

Преобразования дырочных операторов аналогичны. Эти решеточные преобразования приводят гамильтониан (3) к виду

$$
\begin{array}{r}
H=\sum_{\mathbf{k}} \gamma_{\mathbf{k}} \hat{p}_{\mathbf{k}}^{+} \hat{p}_{\mathbf{k}}+\sum_{\mathbf{k}} t_{\mathbf{k}} \hat{h}_{-\mathbf{k}}^{+} \hat{h}_{-\mathbf{k}} \\
-(U / N) \sum_{\mathbf{k} \mathbf{k}^{\prime}} \hat{p}_{\mathbf{k}}^{+} \hat{p}_{\mathbf{k}^{\prime}} \hat{h}_{-\mathbf{k}}^{+} \hat{h}_{-\mathbf{k}^{\prime}},
\end{array}
$$

где $\gamma_{\mathbf{k}}=t_{\mathbf{k}}+U, t_{\mathbf{k}}=-2 t \sum_{\alpha} \cos \left(a k_{\alpha}\right)=t_{-\mathbf{k}}<0-$ затравочный (исходный) потенциал для $n s-30 н, t-$ кинетическая энергия перескока электрона на ближайший узел решетки. В двухчастичной части выражения (4) учтены только те слагаемые в ее точном выражении $-(U / N) \sum_{\mathbf{k k}^{\prime} \mathbf{Q}} \hat{p}_{\mathbf{k}}^{+} \hat{p}_{\mathbf{k}^{\prime}} \hat{h}_{\mathbf{Q}-\mathbf{k}}^{+} \hat{h}_{\mathbf{Q}-\mathbf{k}^{\prime}}$, которые соответствуют членам с $\mathbf{Q}=0$. Тогда гамильтониан Хаббарда сводится к гамильтониану Бардина-Купера-Шриффера (БКШ) [5].

Если учесть вид оператора $\hat{n}$ концентрации электронов исходной системы

$$
\begin{aligned}
\hat{n} \equiv\left(N_{e} / N\right) & =(1 / N) \sum_{j}\left(\hat{n}_{j \uparrow}+\hat{n}_{j \downarrow}\right) \\
& =(1 / N) \sum_{j}\left(\hat{p}_{j}^{+} \hat{p}_{j}+\hat{h}_{j} \hat{h}_{j}^{+}\right)
\end{aligned}
$$

(где $N_{e}-$ полное число электронов в решетке, $N-$ число ее узлов), т.е. формулу

$$
\begin{aligned}
(1 / N) \sum_{j}\left(\hat{p}_{j}^{+} \hat{p}_{j}-\hat{h}_{j}^{+} \hat{h}_{j}\right) & =(1 / N) \sum_{\mathbf{k}}\left(\hat{p}_{\mathbf{k}}^{+} \hat{p}_{\mathbf{k}}-\hat{h}_{-\mathbf{k}}^{+} \hat{h}_{-\mathbf{k}}\right) \\
& =\hat{n}-1,
\end{aligned}
$$

то видно, что в случае половинного $(n=1)$ заполнения исходной затравочной зоны $t_{\mathbf{k}}$ электронами правая часть этой формулы обращается в нуль, и тогда первые два члена гамильтониана (4) можно объединить

$$
\begin{gathered}
H=\sum_{\mathbf{k}}\left(t_{\mathbf{k}}+U / 2\right)\left(\hat{p}_{\mathbf{k}}^{+} \hat{p}_{\mathbf{k}}+\hat{h}_{-\mathbf{k}}^{+} \hat{h}_{-\mathbf{k}}\right) \\
-(U / N) \sum_{\mathbf{k} \mathbf{k}^{\prime}} \hat{p}_{\mathbf{k}}^{+} \hat{p}_{\mathbf{k}^{\prime}} \hat{h}_{-\mathbf{k}}^{+} \hat{h}_{-\mathbf{k}^{\prime}} .
\end{gathered}
$$

В этом случае число электронов равно числу дырок, a выражение (5) принимает точный вид гамильтониана 
БКШ, но в качестве „пары“ здесь выступают электрон и дырка с одинаковым направлением спинов (имея теперь единичный спин и нулевой электрический заряд, экситон фактически является нейтральным бозоном в отличие от случая заряженных куперовских пар с зарядом $q=2 e$, создающих сверхпроводящий ток). Отметим, что элементарные комплексы, способные создавать сверхпроводимость или сверхтекучесть, обязательно относятся к бозонным образованиям: так, в случае сверхпроводимости спин электронной пары Купера равен нулю, в случае сверхтекучести спин атомов гелия ${ }_{4} \mathrm{He}^{2}$ также равен нулю, спин же экситона частица-дырка равен единице, т. е. всегда формируются только целочисленные (бозонные) значения спина.

Половинное заполнение $4 s$-зоны (электронная конфигурация отдельного атома кальция имеет вид $\ldots 3 d^{0} 4 s^{2}$ ) с переходом одного $4 s$-электрона на $3 d$-уровень, по-видимому, возможно как раз в области давлений $32 \leq P \leq 80 \mathrm{GPa}$, т.е. в случае существования именно ПК-решетки.

Для проведения численных расчетов в случае половинного заполнения исходной $n s$-зоны электронами, гамильтониан (5) удобно переписать в виде

$$
H=\sum_{\mathbf{k}}\left(t_{\mathbf{k}}+U / 2\right)\left(\hat{p}_{\mathbf{k}}^{+} \hat{p}_{\mathbf{k}}+\hat{h}_{-\mathbf{k}}^{+} \hat{h}_{-\mathbf{k}}\right)-U \hat{\Delta}^{+} \hat{\Delta}, \quad U>0 .
$$

Здесь введены обозначения для полных операторов экситонов

$$
\hat{\Delta} \equiv(1 / \sqrt{N}) \sum_{\mathbf{k}} \hat{h}_{-\mathbf{k}} \hat{p}_{\mathbf{k}}, \quad \hat{\Delta}^{+} \equiv(1 / \sqrt{N}) \sum_{\mathbf{k}} \hat{p}_{\mathbf{k}}^{+} \hat{h}_{-\mathbf{k}}^{+},
$$

где выражения $\hat{p}_{\mathbf{k}}^{+} \hat{h}_{-\mathbf{k}}^{+} \equiv\left(\hat{b}_{\mathbf{k}}^{+}\right)_{\mathrm{ex}}, \hat{h}_{-\mathbf{k}} \hat{p}_{\mathbf{k}} \equiv\left(\hat{b}_{k}\right)_{\mathrm{ex}}$ суть сами операторы экситонов, выражение $\hat{\Delta}^{+} \hat{\Delta}$ есть оператор полного числа экситонов, а $U-$ энергия образования экситонов.

Для сравнения модельный гамильтониан БКШ имеет вид [5]

$$
\begin{gathered}
H_{\mathrm{BCS}}=\sum_{\mathbf{k}} \varepsilon_{\mathbf{k}}\left(\hat{n}_{k}+\hat{n}_{-k}\right)+V \hat{\Delta}^{+} \hat{\Delta}, \\
\hat{n}_{k} \equiv \hat{C}_{k}^{+} \hat{C}_{k}, \quad \hat{n}_{-k} \equiv \hat{C}_{-k}^{+} \hat{C}_{-k}, \quad V<0, \\
\hat{\Delta} \equiv(1 / \sqrt{N}) \sum_{\mathbf{k}} \hat{C}_{-\mathbf{k} \downarrow} \hat{C}_{\mathbf{k} \uparrow} \equiv(1 / \sqrt{N}) \sum_{\mathbf{k}} \hat{C}_{-k} \hat{C}_{k} \\
\equiv(1 / \sqrt{N}) \sum_{\mathbf{k}} \hat{b}_{k}, \quad \hat{b}_{k}^{+} \equiv \hat{C}_{k}^{+} \hat{C}_{-k}^{+},
\end{gathered}
$$

т. е. в случае $n=1$ существует полная аналогия с гамильтонианом БКШ (где также имелось в виду половинное заполнение $n s$-зоны), $\hat{b}_{k}^{ \pm} \equiv \hat{C}_{ \pm k}^{ \pm} \hat{C}_{\mp k}^{ \pm}$суть операторы пар Купера, а выражение $\hat{\Delta}^{+} \hat{\Delta}$ здесь есть оператор полного их числа.

В гамильтониане (6) перестроенные затравочные зоны $\left(t_{\mathbf{k}}+U / 2\right)$ системы свободных электронов и дырок одинаковы, но выше энергий $t_{\mathbf{k}}$ электронов до их перестройки.
Разумеется, и общий гамильтониан (4) можно записать через операторы экситонов

$$
H=\sum_{\mathbf{k}} \gamma_{\mathbf{k}} \hat{p}_{\mathbf{k}}^{+} \hat{p}_{\mathbf{k}}+\sum_{\mathbf{k}} t_{\mathbf{k}} \hat{h}_{-\mathbf{k}}^{+} \hat{h}_{-\mathbf{k}}-U \hat{\Delta}^{+} \hat{\Delta} .
$$

Однако возможная физическая интерпретация этого общего гамильтониана и численные расчеты с его использованием в настоящей работе приводиться не будут.

\section{3. Вычисление спектров элементарных возбуждений и корреляционных функций}

Вначале находятся уравнения движения (УД) операторов задачи $\hat{A}(t) \equiv \exp (i H t) \hat{A} \exp (-i H t)$, т. е. производная этого оператора по времени оказывается равной его коммутатору с гамильтонианом системы [5]. При этом обычно получаются бесконечные цепочки уравнений, которые приходится обрывать, чтобы получить, как выражался А.А. Власов, математический аппарат, т. е. получить УД в замкнутом виде. Для этой цели и разработан точный (на каждом шаге расцеплений) аналитический метод - прямой алгебраический метод (ПАМ) обрыва цепи УД [5]. Однако в данном случае гораздо проще воспользоваться приближенным расцеплением, которое предложил Валатин [6]. Это расцепление, как известно, позволило ему буквально на половине страницы получить все основные результаты теории БКШ для основного состояния сверхпроводника [6].

Если УД получены, то найдена и $K$-матрица задачи основная матрица метода ПАМ. Тогда можно с помощью ПАМ вычислить, во-первых, спектры элементарных возбуждений и, во-вторых, сами корреляционные функции (КФ) решаемой задачи. Так, основные УД задачи (4), найденные на основе обобщенных расцеплений Валатина, имеют следующий вид:

$$
\begin{gathered}
{\left[\hat{p}_{\mathbf{k}}, H\right]_{-} \approx \gamma_{\mathbf{k}} \hat{p}_{\mathbf{k}}-U \Delta \hat{h}_{-\mathbf{k}}^{+} ; \quad\langle\hat{\Delta}\rangle \equiv \Delta,} \\
{\left[\hat{h}_{-\mathbf{k}}^{+}, H\right]_{-} \approx-U \Delta^{*} \hat{p}_{\mathbf{k}}-t_{\mathbf{k}} \hat{h}_{-\mathbf{k}}^{+} ; \quad\left\langle\hat{\Delta}^{+}\right\rangle \equiv \Delta^{*} .}
\end{gathered}
$$

Здесь базисными операторами служат операторы $\hat{p}_{\mathbf{k}}$ и $\hat{h}_{-\mathbf{k}}^{+}$(можно напомнить, что в теории БКШ эту роль играют операторы $\hat{C}_{\mathbf{k} \uparrow}$ и $\left.\hat{C}_{-\mathbf{k} \downarrow}\right)$, тогда $K$-матрица задачи (4) такова:

$$
K(0 \leq n \leq 2) \equiv K=\left\|\begin{array}{cc}
\gamma_{\mathbf{k}} & -U \Delta \\
K_{12}^{*} & -t_{\mathbf{k}}
\end{array}\right\| .
$$

Для случая частной задачи, т.е. при $n=1$, она имеет вид

$$
K(n=1) \equiv \bar{K}=\left\|\begin{array}{cc}
\gamma_{\mathbf{k}}-U / 2 & -U \Delta \\
K_{12}^{*} & -K_{11}
\end{array}\right\| .
$$

Таким образом, след этой матрицы, как и в теории БКШ, равен нулю. 
При вычислении УД (7) использовались следующие коммутаторы и тождества:

$$
\begin{aligned}
& {\left[\hat{p}_{\mathbf{k}}^{+}, \hat{\Delta}^{+}\right]_{-}=\hat{h}_{-\mathbf{k}}^{+}, \quad\left[\hat{h}_{-\mathbf{k}}^{+}, \hat{\Delta}\right]_{-}=\hat{p}_{\mathbf{k}},} \\
& {\left[\hat{p}_{\mathbf{k}}, \hat{\Delta}\right]_{-}=\left[\hat{h}_{-\mathbf{k}}^{+}, \hat{\Delta}^{+}\right]_{-}=0,} \\
& {[\hat{A}, \hat{B}]_{\mp} \equiv 2 \hat{A} \hat{B}-[\hat{A}, \hat{B}]_{ \pm} ;} \\
& {[\hat{1}, \hat{2} \hat{3}]_{\mp} \equiv \hat{2}[\hat{1}, \hat{3}]_{\mp}+[\hat{1}, \hat{2}]_{-} \hat{3} \text {; }} \\
& {[\hat{1} \hat{2}, \hat{3}]_{\mp} \equiv \hat{1}[\hat{2}, \hat{3}]_{\mp} \pm[\hat{1}, \hat{3}]_{-} \hat{2} \text {. }}
\end{aligned}
$$

В случае гамильтониана (4) (либо (5)) возможные одночастичные спектры элементарных возбуждений (экситонов) с положительной энергией, вычисленные с помощью ПАМ (они суть собственные значения $K$-матрицы для УД (7) и находятся из ее векового уравнения $\lambda^{2}-\lambda \operatorname{Sp} K+\operatorname{det} K=0$ [5]), имеют вид

$\varepsilon_{\mathbf{k}}=(1 / 2)\left\{\operatorname{Sp} K \pm\left[\operatorname{Sp}^{2} K-4 \operatorname{det} K\right]^{1 / 2}\right\} \equiv(1 / 2)\{\operatorname{Sp} K \pm R\}$,

$$
\begin{gathered}
R=2 U \sqrt{\left(\frac{1}{2}+\frac{t_{\mathbf{k}}}{U}\right)^{2}+|\Delta|^{2}} \equiv 2 U R^{\prime}, \\
\varepsilon_{\mathbf{k}}=\left[\frac{1-\delta_{n 1}}{2} \pm R^{\prime}\right] U .
\end{gathered}
$$

В спектрах (10) имеется щель $4 K_{12} K_{21}=4 U^{2}|\Delta|^{2}$ (в выражении для $\varepsilon_{\mathbf{k}}$ следует оставить только знак „Плюс“ перед $R^{\prime}$, так как нас интересуют возбуждения с положительной энергией; кроме того, при $U=0$ должно выполняться условие $\varepsilon_{\mathbf{k}} \rightarrow t_{\mathbf{k}}$ ).

Теперь можно перейти к вычислению КФ задачи (4). Согласно ПАМ [5], это делается с помощью ее $F$-матрицы [5]. В том случае, когда $K$-матрица изучаемой задачи имеет второй порядок (как матрица (8) или (9)), матричные элементы ее $F$-матрицы таковы [5]:

$$
\begin{gathered}
\exp (\beta \operatorname{Sp} K / 2) F_{11}=\operatorname{ch}(\beta R / 2)+Q \operatorname{sh}(\beta R / 2), \\
\exp (\beta \operatorname{Sp} K / 2) F_{12}=-2 R^{-1} K_{12} \operatorname{sh}(\beta R / 2), \\
\exp (\beta \operatorname{Sp} K / 2) F_{21}=-2 R^{-1} K_{21} \operatorname{sh}(\beta R / 2), \\
\exp (\beta \operatorname{Sp} K / 2) F_{22}=\operatorname{ch}(\beta R / 2)-Q \operatorname{sh}(\beta R / 2) .
\end{gathered}
$$

Здесь $Q \equiv\left(K_{22}-K_{11}\right) R^{-1}$, причем [7]

$$
\operatorname{Sp} F=2 \exp (-\beta \operatorname{Sp} K / 2) \operatorname{ch}(\beta R / 2)
$$

$\operatorname{det} F=\exp (-\beta \operatorname{Sp} K)=\exp \left[-\beta U\left(1-\delta_{n 1}\right)\right], \quad \beta \equiv 1 / k T$,

где $k \approx 1.38 \cdot 10^{-23} \mathrm{~J} / \mathrm{K} \approx 0.86 \cdot 10^{-4} \mathrm{eV} / \mathrm{K}$ - постоянная Больцмана.

Вычисление конкретных КФ естественно начать с КФ

$$
\begin{aligned}
\Delta & \equiv(1 / \sqrt{N}) \sum_{\mathbf{k}}\left\langle\hat{h}_{-\mathbf{k}} \hat{p}_{\mathbf{k}}\right\rangle=(1 / \sqrt{N}) \sum_{j}\left\langle\hat{h}_{j} \hat{p}_{j}\right\rangle \\
& =(1 / \sqrt{N}) \sum_{j}\left\langle\hat{C}_{j \downarrow}^{+} \hat{C}_{j \uparrow}\right\rangle \exp \left(i \mathbf{q} \mathbf{R}_{\mathbf{i}}\right)
\end{aligned}
$$

(т. е. щели). Эту КФ (ею обычно описывают магнитные свойства изучаемой системы) легко вычислить из системы двух алгебраических уравнений для КФ $\left\langle\hat{h}_{-\mathbf{k}} \hat{p}_{\mathbf{k}}\right\rangle$ и $\left\langle\hat{h}_{-\mathbf{k}}^{+} \hat{h}_{-\mathbf{k}}\right\rangle$ с помощью ПАМ [5]. Они равны

$$
\begin{gathered}
\left\langle\hat{h}_{-\mathbf{k}} \hat{p}_{\mathbf{k}}\right\rangle=F_{12} / \operatorname{inv} F, \\
\left\langle\hat{h}_{-\mathbf{k}}^{+} \hat{h}_{-\mathbf{k}}\right\rangle=\left(1+F_{11}\right) / \operatorname{inv} F .
\end{gathered}
$$

Здесь

$$
\begin{gathered}
\operatorname{inv} F \equiv 1+\operatorname{Sp} F+\operatorname{det} F \\
\langle\ldots\rangle \equiv \operatorname{Sp}[\exp (-\beta H) \ldots] / \operatorname{Sp}[\exp (-\beta H)] .
\end{gathered}
$$

Уравнения для КФ легко написать с помощью ПАМ [5], имея в виду базисные операторы $\hat{p}_{\mathbf{k}}$ и $\hat{h}_{-\mathbf{k}}^{+}$рассматриваемой задачи (см. УД (7)).

Например, уравнение для первой КФ $\left\langle\hat{h}_{-\mathbf{k}} \hat{p}_{\mathbf{k}}\right\rangle$

$$
\left\langle\hat{h}_{-\mathbf{k}} \hat{p}_{\mathbf{k}}\right\rangle=\left\langle\hat{p}_{\mathbf{k}}(\beta) \hat{h}_{-\mathbf{k}}\right\rangle=F_{11}\left\langle\hat{p}_{\mathbf{k}} \hat{h}_{-\mathbf{k}}\right\rangle+F_{12}\left\langle\hat{h}_{-\mathbf{k}}^{+} \hat{h}_{-\mathbf{k}}\right\rangle,
$$

где $\hat{A}[\beta] \equiv \exp (\beta H) \hat{A} \exp (-\beta H)$ - „одетый“ оператор, он не равен оператору $\hat{A}(t)$.

Аналогичным образом пишется уравнение и для второй КФ $\left\langle\hat{h}_{-\mathbf{k}}^{+} \hat{h}_{-\mathbf{k}}\right\rangle$

$$
\begin{aligned}
\left\langle\hat{h}_{-\mathbf{k}}^{+} \hat{h}_{-\mathbf{k}}\right\rangle & =1-\left\langle\hat{h}_{-\mathbf{k}} \hat{h}_{-\mathbf{k}}^{+}\right\rangle=1-\left\langle\hat{h}_{-\mathbf{k}}^{+}(\beta) \hat{h}_{-\mathbf{k}}\right\rangle \\
& =1-F_{21}\left\langle\hat{p}_{\mathbf{k}} \hat{h}_{-\mathbf{k}}\right\rangle+F_{22}\left\langle\hat{h}_{-\mathbf{k}}^{+} \hat{h}_{-\mathbf{k}}\right\rangle .
\end{aligned}
$$

Решения этих двух уравнений - выражения (12) и (13). Для случая операторного базиса большей мерности (трех-, четырехмерного и т.д.) уравнения пишутся по аналогичной схеме (см. в [5] раздел 2.4, формула (2.4.1)).

В случае альтернантных кристаллических решеток удобно сразу прибегнуть к масштабному преобразованию вида $k_{\alpha} \rightarrow \bar{k}_{\alpha} \equiv a k_{\alpha}$ (где $\left.\alpha=1, \ldots, D\right)$, при котором исходные интегралы

$$
\begin{aligned}
(1 / N) \sum_{\mathbf{k}}(\ldots) & \rightarrow \int_{\omega_{\mathbf{k}}}\left(d \mathbf{k} /\left|\omega_{\mathbf{k}}\right|\right)(\ldots) \\
& \equiv \prod_{\alpha=1}^{D}\left[\int_{-\pi / a}^{\pi / a}\left(d k_{\alpha} /(2 \pi / a)\right)\right](\ldots)
\end{aligned}
$$

(где $\omega_{\mathbf{k}}-$ собственно ячейка, т. е. первая зона Бриллюэна, которая для этих типов решеток задается условиями $-(\pi / a) \leq k_{\alpha} \leq(\pi / a)$ и имеет объем $\left.\left|\omega_{\mathbf{k}}\right|=(2 \pi / a)^{D}\right)$ переходят в безразмерные интегралы

$$
\prod_{\alpha=1}^{D}\left[\int_{-\pi}^{\pi}\left(d \bar{k}_{\alpha} / 2 \pi\right)(\ldots)\right]=\prod_{\alpha=1}^{D}\left[\int_{0}^{\pi}\left(d \bar{k}_{\alpha} / \pi\right)\right](\ldots)
$$

по ячейке $-\pi \leq \bar{k}_{\alpha} \leq \pi$ с объемом $(2 \pi)^{D}$.

Используя матричные элементы $(11) F$-матрицы, можно из КФ $\left\langle\hat{h}_{-\mathbf{k}} \hat{p}_{\mathbf{k}}\right\rangle$ извлечь уравнение для щели $\Delta \equiv S$ (щель $S$ входит в выражение для $R^{\prime}$ )

$$
1=\frac{1}{2 N} \sum_{\mathbf{k}} \frac{1}{R^{\prime}}\left\{\frac{\operatorname{sh}\left(\theta R^{\prime}\right)}{\operatorname{ch}[(\beta \operatorname{Sp} K) / 2]+\operatorname{ch}\left(\theta R^{\prime}\right)}\right\}
$$


и уравнение для критической безразмерной температуры $\theta_{c} \equiv \beta_{c} U=U / k T_{c}$

$$
1=\frac{1}{2 N} \sum_{\mathbf{k}} \frac{1}{\left|\xi_{\mathbf{k}}\right|}\left\{\frac{\operatorname{sh}\left(\theta_{c}\left|\xi_{\mathbf{k}}\right|\right)}{\operatorname{ch}\left[\left(\beta_{c} \operatorname{Sp} K\right) / 2\right]+\operatorname{ch}\left(\theta_{c}\left|\xi_{\mathbf{k}}\right|\right)}\right\},
$$

которое получается из (14) в случае, когда щель $S$ обращается в нуль.

Здесь приняты следующие обозначения: $\theta \equiv \beta U$ безразмерная температура, $R^{\prime} \equiv\left(\xi_{\mathbf{k}}^{2}+S^{2}\right)^{1 / 2}$,

$$
\xi_{\mathbf{k}} \equiv(1 / 2)+\left(t_{\mathbf{k}} / U\right)=(1 / 2)-2 \kappa \sum_{\alpha=1}^{D} \cos \left(a k_{\alpha}\right)
$$

- безразмерная затравочная зона, $a$ - постоянная решетки, $D$ - мерность решетки (рассматриваются одномерная, двумерная и трехмерная решетки), $\kappa \equiv t / U$. Формулы (14) и (15) записаны для общего случая заполнения исходной $n s$-зоны $0 \leq n \leq 2$.

\section{4. Вычисление энергии кристалла}

ПАМ позволяет очень просто вычислить и любые другие КФ, например те, которые нужны для расчета энергии изучаемой системы. Первая система уравнений пишется для КФ $\left\langle\hat{p}_{\mathbf{k}}^{+} \hat{p}_{\mathbf{k}}\right\rangle$ и $\left\langle\hat{h}_{-\mathbf{k}}^{+} \hat{p}_{\mathbf{k}}^{+}\right\rangle$. Ее решение для первой из этих двух КФ есть [5]

$$
\sum_{\mathbf{k}}\left\langle\hat{p}_{\mathbf{k}}^{+} \hat{p}_{\mathbf{k}}\right\rangle=\sum_{\mathbf{k}}\left[\operatorname{det} F+F_{11}\right] / \operatorname{inv} F=\left\langle\hat{n}_{j \uparrow}\right\rangle \equiv n_{\uparrow} .
$$

КФ $\left\langle\hat{h}_{-\mathbf{k}}^{+} \hat{p}_{\mathbf{k}}^{+}\right\rangle$далее не понадобится.

Вторая система уравнений пишется для следующих двух КФ: $\left\langle\hat{\Delta}^{+} \hat{h}_{-\mathbf{k}} \hat{p}_{\mathbf{k}}\right\rangle$ и $\left\langle\hat{\Delta}^{+} \hat{h}_{-\mathbf{k}} \hat{h}_{-\mathbf{k}}^{+}\right\rangle$. Решение этой системы для первой из указанных КФ (вторая КФ опять не используется) есть [5]

$$
\begin{gathered}
\left\langle\hat{\Delta}^{+} \hat{h}_{-\mathbf{k}} \hat{p}_{\mathbf{k}}\right\rangle=\sigma_{1} / \sigma, \\
\sigma=\operatorname{inv} F \\
=2 \exp \left(-\frac{\beta \operatorname{Sp} K}{2}\right)\left[\operatorname{ch}\left(\frac{\beta \operatorname{Sp} K}{2}\right)+\operatorname{ch}\left(\beta U R^{\prime}\right)\right] \neq 0, \\
\sigma_{1}=\left(F_{11}+\operatorname{det} F\right)\left\langle h_{-\mathbf{k}}^{+} h_{-\mathbf{k}}\right\rangle+\Delta^{*} F_{12} .
\end{gathered}
$$

Здесь во избежание путаницы обычно используемые в формулах Крамера греческие буквы $\Delta, \Delta_{1}, \Delta_{2}, \ldots$ заменены на греческие буквы $\sigma, \sigma_{1}, \sigma_{2}, \ldots$ (такая замена делается только в формулах Крамера, не путать их со спиновыми переменными $\sigma$ ).

Первая КФ позволяет получить четырехоператорную часть гамильтониана (4). Действительно, можно после- довательно записать

$$
\begin{aligned}
-U\left\langle\Delta^{+} \Delta\right\rangle= & -(U / \sqrt{N}) \sum_{\mathbf{k}}\left\langle\hat{\Delta}^{+} \hat{h}_{-\mathbf{k}} \hat{p}_{\mathbf{k}}\right\rangle \\
= & -(U / 4) \sum_{\mathbf{k}}\left\{(1 / N)\left[\exp (-\beta \operatorname{Sp} K / 2)+\Phi_{1}\right]\right. \\
& \times\left[\exp (-\beta \operatorname{Sp} K / 2)+\Phi_{1}\right] \\
& \left.+\left(2|\Delta|^{2} / R^{\prime}\right) \Phi_{2} \operatorname{sh}\left(\beta U R^{\prime}\right)\right\} / \Phi_{2}^{2}
\end{aligned}
$$

где

$$
\begin{gathered}
\Phi_{1} \equiv \operatorname{ch}\left(\beta U R^{\prime}\right)+Q \operatorname{sh}\left(\beta U R^{\prime}\right), \\
\Phi_{2} \equiv \operatorname{ch}(\beta \operatorname{Sp} K / 2)+\operatorname{ch}\left(\beta U R^{\prime}\right) .
\end{gathered}
$$

Теперь можно получить общее выражение для искомой энергии, приходящейся на одну ячейку, которая есть среднее значение, например, гамильтониана (4) для случая $0 \leq n \leq 2$ :

$$
\begin{aligned}
E= & \langle H / N\rangle \\
= & (1 / N)\left[U \sum_{\mathbf{k}}\left\langle\hat{p}_{\mathbf{k}}^{+} \hat{p}_{\mathbf{k}}\right\rangle+\sum_{\mathbf{k}} t_{\mathbf{k}}\left(\left\langle\hat{p}_{\mathbf{k}}^{+} \hat{p}_{\mathbf{k}}\right\rangle+\left\langle\hat{h}_{-\mathbf{k}}^{+} \hat{h}_{-\mathbf{k}}\right\rangle\right)\right] \\
& -(U / N)\left\langle\hat{\Delta}^{+} \hat{\Delta}\right\rangle .
\end{aligned}
$$

Отдельные слагаемые в этом выражении имеют вид

$$
\begin{aligned}
(1 / N) & \sum_{\mathbf{k}} \gamma_{\mathbf{k}}\left\langle\hat{p}_{\mathbf{k}}^{+} \hat{p}_{\mathbf{k}}\right\rangle=(1 / 2 N) \\
& \times \sum_{\mathbf{k}} \gamma_{\mathbf{k}} \frac{\operatorname{ch}\left(\beta U R^{\prime}\right)+Q \operatorname{sh}\left(\beta U R^{\prime}\right)+\exp (-\beta \operatorname{Sp} K / 2)}{\operatorname{ch}(\beta \operatorname{Sp} K / 2)+\operatorname{ch}\left(\beta U R^{\prime}\right)},
\end{aligned}
$$

где

$$
\begin{gathered}
Q \equiv-\left(\frac{1}{2}+\frac{t_{\mathbf{k}}}{U}\right) / R^{\prime}, \quad \operatorname{Sp} K=U\left(1-\delta_{n 1}\right) \\
(1 / N) \sum_{\mathbf{k}} t_{\mathbf{k}}\left\langle\hat{h}_{-\mathbf{k}}^{+} \hat{h}_{-\mathbf{k}}\right\rangle=(1 / 2 N) \\
\times \sum_{\mathbf{k}} t_{\mathbf{k}} \frac{\operatorname{ch}\left(\beta U R^{\prime}\right)+Q \operatorname{sh}\left(\beta U R^{\prime}\right)+\exp (\beta \operatorname{Sp} K / 2)}{\operatorname{ch}(\beta \operatorname{Sp} K / 2)+\operatorname{ch}\left(\beta U R^{\prime}\right)}, \\
-(U / N)\left\langle\hat{\Delta}^{+} \hat{\Delta}\right\rangle=-(U / 2 N)|\Delta|^{2} \\
\times \sum_{\mathbf{k}} \frac{1}{R^{\prime}} \frac{\operatorname{sh}\left(\beta U R^{\prime}\right)}{\operatorname{ch}(\beta \operatorname{Sp} K / 2)+\operatorname{ch}\left(\beta U R^{\prime}\right)}
\end{gathered}
$$

Из (21) видно, что в момент исчезновения щели $(\Delta=0)$ в энергетическом спектре элементарных возбуждений (экситонов) энергия их образования (21) обращается в нуль. Таким образом, энергетически выгодным для этой бозонной системы оказывается случай $\Delta \neq 0$, поскольку тогда полная энергия понижается из-за отрицательности энергии образования экситонов, хотя кинетическая энергия $\left(t_{\mathbf{k}}+U / 2\right)($ см. $(6))$ свободных электронов и дырок 
в кристаллическом поле у этой системы электронов и дырок теперь выше, чем кинетическая энергия $t_{\mathbf{k}}$ свободных электронов в исходной системе (т.е. в энергетическом плане снова имеется полная аналогия с теорией БКШ).

\section{5. Основное состояние кристалла}

Особое место занимает случай нулевой температуры, соответствующий основному состоянию рассматриваемого кристалла. Для анализа этого случая нужно выяснить пределы гиперболических комбинаций в правых частях уравнений (19)-(21) при $\beta \rightarrow \infty$. Асимптотика этих выражений при условии $(1 / 2)-R^{\prime}<0$ имеет вид

$$
\begin{aligned}
& (1 / N) \sum_{\mathbf{k}} \gamma_{\mathbf{k}}\left\langle\hat{p}_{\mathbf{k}}^{+} \hat{p}_{\mathbf{k}}\right\rangle \approx(1 / 2 N) \sum_{\mathbf{k}}(1+Q) \gamma_{\mathbf{k}}, \\
& (1 / N) \sum_{\mathbf{k}} t_{\mathbf{k}}\left\langle\hat{h}_{-\mathbf{k}}^{+} \hat{h}_{-\mathbf{k}}\right\rangle \approx(1 / 2 N) \sum_{\mathbf{k}}(1+Q) t_{\mathbf{k}}, \\
& -(U / N)\left\langle\hat{\Delta}^{+} \hat{\Delta}\right\rangle \approx-(U / N)|\Delta|^{2}(1 / 2) \sum_{\mathbf{k}}\left(1 / R^{\prime}\right) .
\end{aligned}
$$

В обратном же случае - при $(1 / 2)-R^{\prime}>0$ - выражения (22) и (24) обращаются в нуль, а выражение (23) в этом случае дает среднее значение зонной энергии $(1 / N) \sum_{\mathbf{k}} t_{\mathbf{k}} \equiv\left\langle t_{k}\right\rangle$.

Таким образом, для энергии основного состояния, приходящейся на одну ячейку кристалла, получается выражение

$$
\begin{aligned}
& \langle H / N\rangle_{0} \equiv E_{0} \\
& =U\left\{\prod_{\alpha=1}^{D}\left[\int_{0}^{\bar{k}_{\mathrm{F}}} \frac{d \bar{k}_{\alpha}}{\pi}\right]\left[\frac{1}{2}-2 \kappa\left(\cos \bar{k}_{x}+\cos \bar{k}_{y}+\cos \bar{k}_{z}\right)\right]\right. \\
& \left.\quad \times(1+Q)-|\Delta|^{2} \prod_{\alpha=1}^{D}\left[\int_{0}^{\bar{k}_{\mathrm{F}}} \frac{d \bar{k}_{\alpha}}{\pi}\right] \frac{1}{2 R^{\prime}}\right\}
\end{aligned}
$$

где интегрирование по $\bar{k}_{\alpha}$ ведется до значения $\bar{k}_{\alpha}=\bar{k}_{\mathrm{F}}$ (см. далее).

Здесь величина $|\Delta|^{2} \equiv S^{2}$, т.е. квадрат ширины щели при нулевой температуре, вычисляется из трансцендентного уравнения

$$
\prod_{\alpha=1}^{D}\left[\int_{0}^{\bar{k}_{\mathrm{F}}} \frac{d \bar{k}_{\alpha}}{\pi}\right]\left\{\left[\frac{1}{2}-2 \kappa \sum_{\alpha=1}^{D} \cos \bar{k}_{\alpha}\right]^{2}+|\Delta|^{2}\right\}^{-1 / 2}=2
$$

Если поверхность Ферми есть сфера, то ее радиус дается выражением $k_{\mathrm{F}}=\left[6 \pi^{2} n / g\right]^{1 / 3}$, где $n \equiv N_{e} / V$ $=N_{e} /\left(\left|\omega_{\mathbf{r}}\right| N_{\text {cell }}\right)$ - плотность электронов, $V$ - объем системы, $g=2(2 l+1)$ - кратность вырождения уровня свободного атома, из которого в кристалле образовалась данная энергетическая $n s$-зона, т.е. для $s$-зоны $l=0$, $g=2$. Если далее считать, что на каждый узел (атом) приходится один электрон, то $N_{e} / N_{\text {cell }}=1$, и тогда $n=1 /\left|\omega_{\mathbf{r}}\right|=1 / 8$, так как $\left|\omega_{\mathbf{r}}\right|\left|\omega_{\mathbf{k}}\right|=(2 \pi)^{3}$, a $\left|\omega_{\mathbf{k}}\right|=\pi^{3}$. Тогда $a k_{\mathrm{F}} \equiv \bar{k}_{\mathrm{F}}=\left(3 \pi^{2} / 8\right)^{1 / 3} \approx 1.55$.

\section{6. Численный расчет поведения щели и температуры ее исчезновения}

Поскольку нам неизвестны надежные экспериментальные данные по измерению электросопротивления ПК-фазы кальция (по этому вопросу см. работу [2] и ссылки в ней), мы решили аналитически по нашей методике проверить поведение щели и критической температуры в кристалле кальция с ПК-решеткой, т. е. численно решались многопараметрические трансцендентные уравнения для случая половинного $(\operatorname{Sp} K=0)$ заполнения зоны, а именно уравнение для щели $\Delta=S$ (входит в $R^{\prime} \equiv\left(\xi_{\mathbf{k}}^{2}+S^{2}\right)^{1 / 2}$, см. уравнение $\left.(14)\right)$

$$
\begin{aligned}
1 & =\left.\frac{1}{2 N} \sum_{\mathbf{k}} \frac{1}{R^{\prime}}\left\{\frac{\operatorname{sh}\left(\theta R^{\prime}\right)}{\operatorname{ch}[(\beta \operatorname{Sp} K) / 2]+\operatorname{ch}\left(\theta R^{\prime}\right)}\right\}\right|_{n=1} \\
& =\frac{1}{2 \pi^{D}} \int_{0}^{\pi} d x d y d z \frac{1}{R^{\prime}} \operatorname{th}\left(\frac{1}{2} \theta R^{\prime}\right)
\end{aligned}
$$

и уравнение для критической температуры $\theta_{c} \equiv \beta_{c} U=$ $U / k T_{c}$ (см. уравнение (15))

$$
\begin{aligned}
1 & =\left.\frac{1}{2 N} \sum_{\mathbf{k}} \frac{1}{\left|\xi_{\mathbf{k}}\right|}\left\{\frac{\operatorname{sh}\left(\theta_{c}\left|\xi_{\mathbf{k}}\right|\right)}{\operatorname{ch}\left[\left(\beta_{c} \operatorname{Sp} K\right) / 2\right]+\operatorname{ch}\left(\theta_{c}\left|\xi_{\mathbf{k}}\right|\right)}\right\}\right|_{n=1} \\
& =\frac{1}{2 \pi^{D}} \int_{0}^{\pi} d x d y d z \frac{1}{\left|\xi_{\mathbf{k}}\right|} \operatorname{th}\left(\frac{1}{2} \theta_{c}\left|\xi_{\mathbf{k}}\right|\right) .
\end{aligned}
$$

Для основного состояния кристалла, т.е. для случая $\theta \rightarrow \infty$, формулы (27) и (28) примут соответственно вид (при $\left.(1 / 2)-R^{\prime}<0\right)$

$$
\begin{aligned}
& 1=\frac{1}{2 \pi^{D}} \int_{0}^{x_{\mathrm{F}} y_{\mathrm{F}} z_{\mathrm{F}}} d x d y d z\left(1 / R^{\prime}\right), \\
& 1=\frac{1}{2 \pi^{D}} \int_{0}^{x_{\mathrm{F}} y_{\mathrm{F}} z_{\mathrm{F}}} d x d y d z\left(1 /\left|\xi_{\mathbf{k}}\right|\right) .
\end{aligned}
$$

\section{7. Обсуждение результатов}

Численные расчеты поведения щели и температуры ФП металл-экситонный изолятор по формулам (27) и (28) проводились для одно-, дву- и трехмерных решеток. Результаты этих расчетов представлены на 

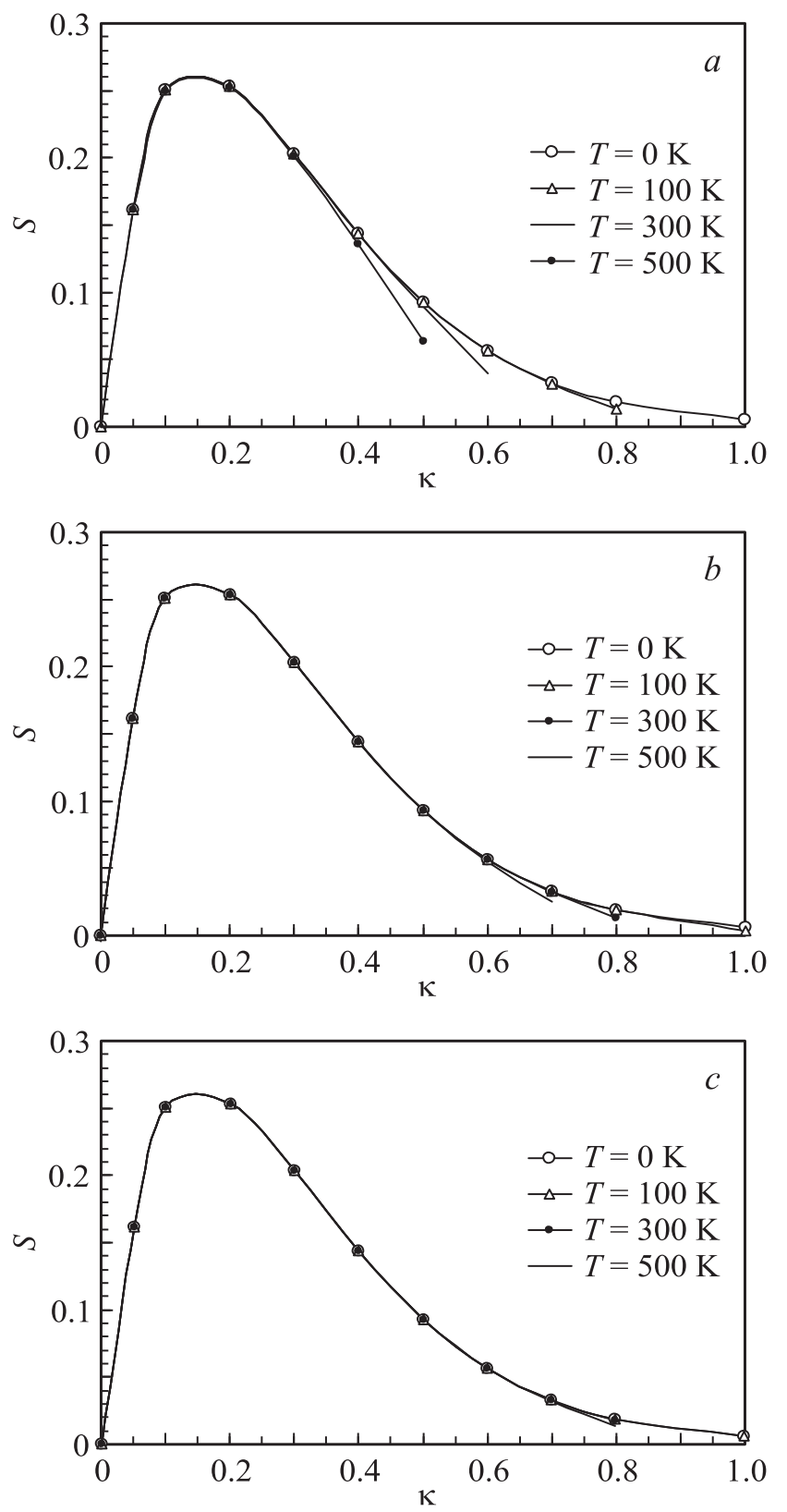

Рис. 1. Зависимости щели $S$ от переменной $\kappa$ для трехмерного кристалла при различных температурах в безразмерных координатах. $U=1(a), 3(b), 5 \mathrm{eV}(c)$.

рис. 1-4. Ошибка численного решения точных уравнений составляет не более половины процента. Щель $S$ и температура перехода $\theta_{c}$ рассматривались в виде двухпараметрических функций (параметры $U$ и $T$ ) одной независимой переменной $\kappa \equiv t / U$, поскольку именно с этой переменной связан фактический учет наличия кристаллической решетки. При $\kappa=0$ вообще нет никакой решетки, а уравнения вырождаются в простые алгебраические соотношения. При $\kappa=1$ кинетическая энергия $t$ (точнее, энергия перескока электрона на ближайший узел) и интенсивность взаимодействия $U$ электронов на одном узле оказываются равными, и поэтому условий

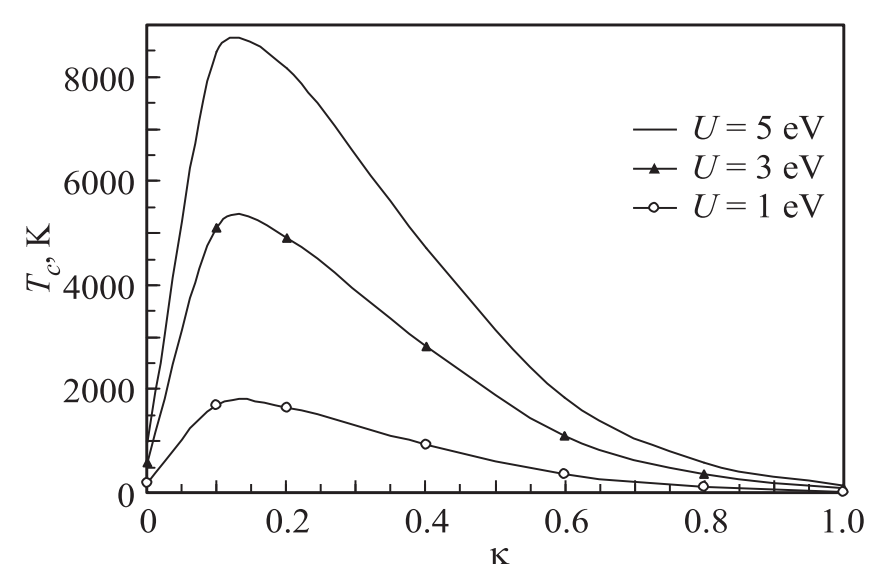

Рис. 2. Зависимость температуры $T_{c}$ исчезновения щели от переменной $\kappa$ для трехмерного кристалла.
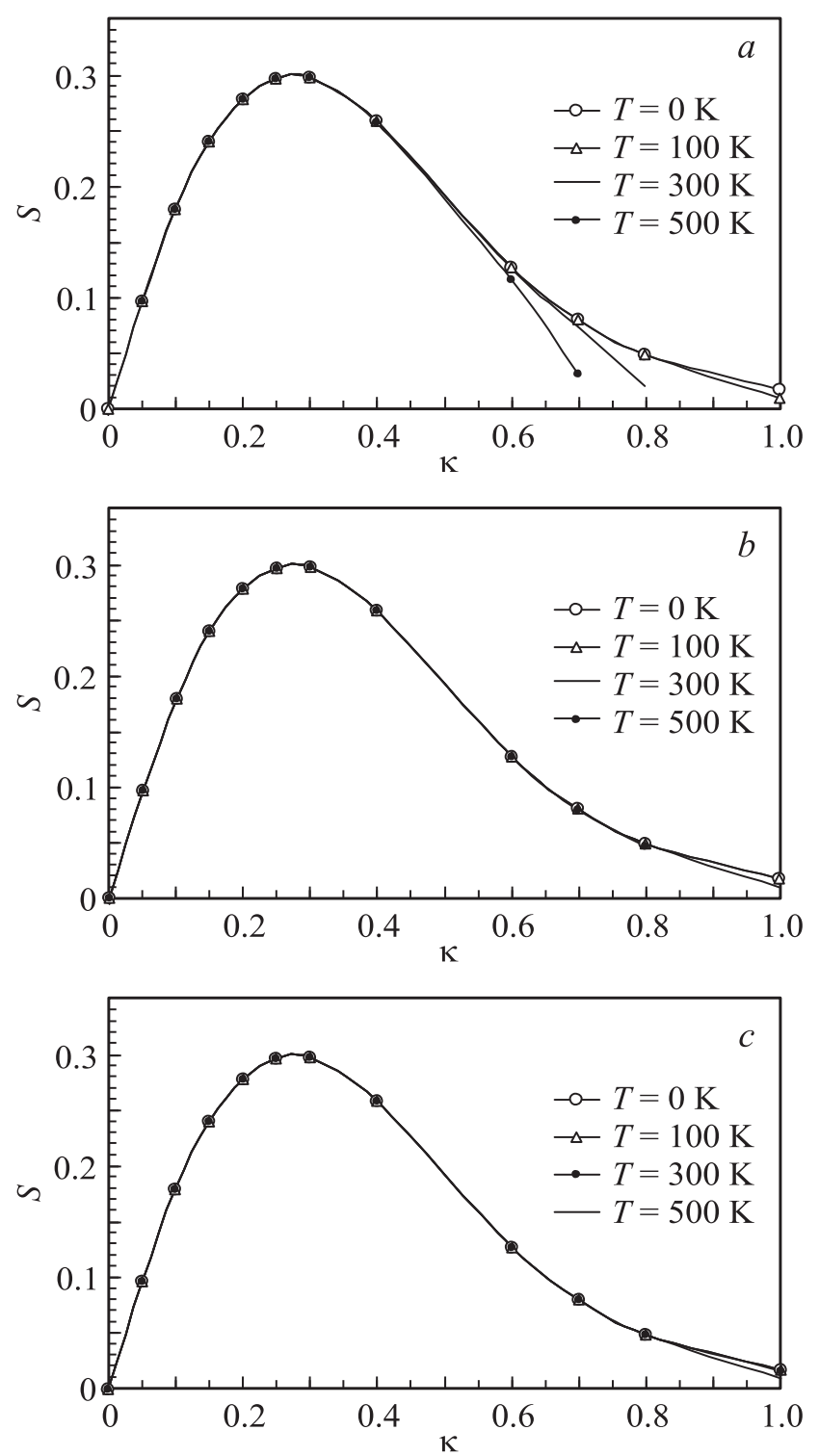

Рис. 3. Зависимости щели $S$ от переменной $\kappa$ для одномерного кристалла при различных температурах в безразмерных координатах. $U=1(a), 3(b), 5 \mathrm{eV}(c)$. 


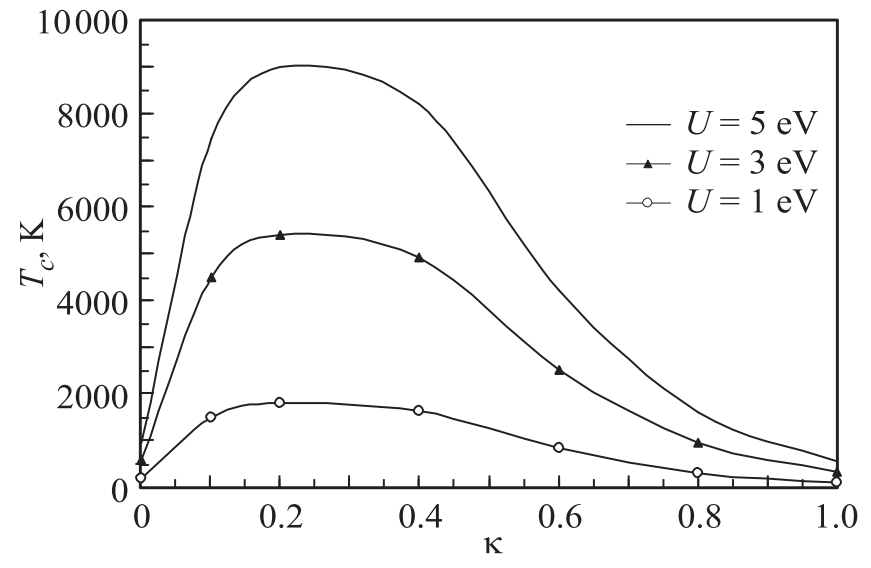

Рис. 4. Зависимость температуры $T_{c}$ исчезновения щели от переменной $\kappa$ для одномерного кристалла.

для ФП снова нет. Значение интеграла перескока $t$ в расчетах бралось равным $0.3 \mathrm{eV}$, а величина взаимодействия $U$ электронов (с разными спинами) на одном узле решетки менялась от 1 до $10 \mathrm{eV}$, но результаты расчетов приведены только для трех значений $U=1,3,5 \mathrm{eV}$. Надежных данных для внутренних параметров $t$ и $U$ модели Хаббарда, насколько нам известно, в литературе нет (см. [2,8-10]). Поэтому мы решили считать $\kappa$ отдельной независимой переменной, изменяющейся в пределах $0 \leq \kappa \leq 1$, а параметр $U$ менять только в аргументе гиперболического тангенса th (...). Таким образом, по нашим аналитическим расчетам, как это видно из рисунков, трехмерный кристалл Са с ПК-решеткой существует только в виде экситонного изолятора при всех температурах, при которых он вообще существует еще в виде кристалла.

\section{8. Заключение}

Поскольку в расчетах не использовались конкретные свойства Са, выводы относительно его перехода в экситонный изолятор должны оставаться справедливыми (разумеется, при соответствующих условиях) и для всех кристаллов с ПК-решеткой (например, для полония $\left.{ }_{209} \mathrm{Po}^{84} \equiv{ }_{A} \mathrm{Po}^{Z}\right)$.

В литературе имеется [11] точный аналитический расчет по поводу возможности существования ФП моттовского типа изолятор-металл в одномерном кристалле, находящемся в основном состоянии и описываемом именно гамильтонианом Хаббарда (с половинным заполнением зон), который показал, что при любых конечных значениях энергии $U$ взаимодействия его электронов в этой системе нет ФП изолятор-металл (это состояние является металлическим лишь при $U=0)$. Мы (с целью проверки согласованности результатов нашей схемы расчетов с результатом точного расчета) провели такой же расчет по нашей методике, но, разумеется, для обратного моттовского ФП металл-изолятор. По нашим расчетам такой кристалл при тех же условиях и $U \neq 0$ остается, как и трехмерный кристалл, экситонным изолятором, что вполне согласуется с выводом работы [11] об отсутствии ФП моттовского типа изолятор-металл.

Расчеты проводились для трехмерного, двумерного и одномерного кристаллов при температурах $0 \leq T \leq 500 \mathrm{~K}$ и $U=1,3,5 \mathrm{eV}$ (значение $U$ изменялось только в аргументе гиперболического тангенса $\operatorname{th}\left(\theta R^{\prime} / 2\right)$, где $\left.\theta \equiv \beta U\right)$. Однако, чтобы не увеличивать объем статьи, графические результаты расчетов для двумерных кристаллов здесь не приводятся.

Значения рассчитываемых трансцендентных интегралов следующие: $2 \pi^{3} \approx 62.012$ - значение трехмерного интеграла, $2 \pi^{2} \approx 19.739$ - значение двумерного интеграла, $2 \pi \approx 6.283$ - значение одномерного интеграла.

Материал работы докладывался на семинаре по теоретической физике в Российском федеральном ядерном центре-Всероссийском научно-исследовательском институте экспериментальной физики (РФЯЦ-ВНИИЭФ).

\section{Список литературы}

[1] J. Hubbard. Proc. Roy. Soc. A 236, 238 (1963).

[2] В.Е. Фортов, А.М. Молодец, В.И. Постнов, Д.В. Шахрай, К.Л. Коган, Е.Г. Максимов, А.В. Иванов, М.В. Магницкая. Письма в ЖЭТФ 79, 425 (2004).

[3] H. Shiba. Prog. Theor. Phys. 48, 2171 (1972).

[4] А.С. Давыдов. Теория твердого тела. Наука, М. (1976). $637 \mathrm{c}$.

[5] М.Ф. Сарры. УФН 161, 11, 47 (1991).

[6] Д. Пайнс. Проблема многих тел. ИИЛ, М. (1963). 191 с.

[7] Р. Фейнман. Статистическая механика. Мир, М. (1978). $408 \mathrm{c}$.

[8] H.L. Skriver. Phys. Rev. Lett. 49, 1768 (1982).

[9] R.A. Stager, H.G. Drickamer. Phys. Rev. 131, 2524 (1963).

[10] K.J. Dunn, F.P. Bundy. Phys. Rev. B 24, 1643 (1981).

[11] E.H. Lieb, F.Y. Wu. Phys. Rev. Lett. 20, 1445 (1968). 